\title{
Larsen-like osseous dysplasia-short stature syndrome
}

INSERM

\section{Source}

INSERM. (1999). Orphanet: an online rare disease and orphan drug data base. Larsen-like osseous dysplasia-short stature syndrome. ORPHA:2370

Larsen-like osseous dysplasia-short stature syndrome is a rare primary bone dysplasia characterized by a Larsen-like phenotype including multiple, congenital, large joint dislocations, craniofacial abnormalities (i.e. macrocephaly, flat occiput, prominent forehead, hypertelorism, low-set, malformed ears, flat nose, cleft palate), spinal abnormalities, cylindrical fingers, and talipes equinovarus, as well as growth retardation (resulting in short stature) and delayed bone age. Other reported clinical manifestations include severe developmental delay, hypotonia, clinodactyly, congenital heart defect and renal dysplasia. 